\title{
Development of a novel PIG-A gene mutation assay based on a GPI-anchored fluorescent protein sensor
}

\author{
Xu Tian', Youjun Chen ${ }^{2}$ and Jun Nakamura ${ }^{1,3^{*}}$
}

\begin{abstract}
Background: Accumulation of somatic mutations caused by both endogenous and exogenous exposures is a high risk for human health, in particular, cancer. Efficient detection of somatic mutations is crucial for risk assessment of different types of exposures. Due to its requirement in the process of attaching glycosylphatidylinositol- (GPI-) anchored proteins to the cell surface, the PIG-A gene located on the X-chromosome is used in both in vivo and in vitro mutation assays. Loss-of-function mutations in PIG-A lead to the elimination of GPI-anchored proteins such that they can no longer be detected on the cell surface by antibodies. Historically, mutation assays based on the PIG-A gene rely on the staining of these cell-surface proteins by antibodies; however, as with any antibody-based assay, there are major limitations, especially in terms of variability and lack of specific antibodies.

Results: In the current study, we developed a modified PIG-A mutation assay that uses the expression of GPIanchored fluorescent proteins (henceforth referred to as a GPI-sensor), whereby the presence of fluorescence on the cell membrane is dependent on the expression of wild-type PIG-A. Using our modified PIG-A mutation assay, we have achieved complete separation of wild type cells and spontaneously mutated cells, in which the presence of PIG-A mutations has been confirmed via proaerolysin resistance and gene sequencing.
\end{abstract}

Conclusion: This study establishes a novel PIG-A mutation assay using GPI-anchored fluorescent protein expression that eliminates the need for antibody-based staining. This GPI-sensor PIG-A mutation assay should be widely applicable for accurate and efficient testing of genotoxicity for use in many mammalian and vertebrate cells.

Keywords: PIG-A, GPI-sensor, Fluorescent protein, Genotoxicity, Gene mutation assay, Flow cytometry

\section{Introduction}

The accumulation of somatic mutations due to both endogenous and exogenous chemical exposures has been long known to be dangerous for human health and to cause cancer [1]. Efficient detection of somatic mutations is crucial for risk assessment of different types of exposures and is of great interest to many scientists, ranging from basic researchers to regulatory specialists. In particular, the flow cytometry-based PIG- $A$ mutation assay is a commonly-used method used to detect mutations that

\footnotetext{
* Correspondence: junnakamura77777@gmail.com

'Department of Environmental Sciences and Engineering, University of North Carolina at Chapel Hill, Chapel Hill, NC, USA

${ }^{3}$ Laboratory of Laboratory Animal Science, Graduate School of Life and Environmental Biosciences, Osaka Prefecture University, Izumisano, Osaka, Japan

Full list of author information is available at the end of the article
}

develop in the PIG-A gene, which is necessary for the glycosylphatidylinositol (GPI) anchor biosynthesis pathway, by assaying for the presence of cell-surface GPIanchored proteins [2].

The GPI anchor is a class of glycolipid structures that anchor $10-20 \%$ of cell-surface proteins to the plasma membrane and is synthesized in the endoplasmic reticulum in a pathway consisting of 11 cascading steps. A total of 22 GPI anchor synthesis proteins (GASPs) are involved in GPI anchor synthesis, such as PIG-A, PIG-C, PIG-K, PIG-O, and PIG-S, among others [3]. The PIG-A gene is the only GASP encoded by a gene located on the $\mathrm{X}$-chromosome and encodes a critical GASP responsible for catalyzing the first step of GPI anchor synthesis [4]. In fact, the function of PIG-A was first characterized after the identification of a mutation in patients with paroxysmal nocturnal hemoglobinuria $[5,6]$. In these

(c) The Author(s). 2019 Open Access This article is distributed under the terms of the Creative Commons Attribution 4.0 International License (http://creativecommons.org/licenses/by/4.0/), which permits unrestricted use, distribution, and 
patients, loss-of-function mutations in PIG-A result in the elimination of cell-surface GPI-anchored proteins, leading to the destruction of red blood cells by the complement system and ultimately intravascular hemolytic anemia [5-7].

More specifically, GPI-anchored proteins are first synthesized as precursors. The N-terminal hydrophobic signal peptide (N-SP) targets the newly-synthesized protein to the endoplasmic reticulum lumen where the $\mathrm{N}$ $\mathrm{SP}$ is cleaved by a signal peptidase. The C-terminal GPI anchor signal sequence (C-SP) is also cleaved, and the resulting mature protein is attached to the GPI-anchor by an amide bond via a transamidase [8]. In the absence of GPI-anchor synthesis, the resulting unanchored proteins cannot be targeted to the cell membrane. In this situation, the destination of the protein from the endoplasmic reticulum depends on the status of the GPI anchor synthesis. If GPI anchor synthesis is blocked prior to the incorporation of the first mannose (for example, in PIG-A deficient mammalian cells), the hydrophobic C-SP will not be cleaved and the protein will be recognized as an unfolded protein and degraded by the proteasomal degradation pathway. If GPI anchor synthesis is blocked after the incorporation of the mannose (for example in PIG-O deficient chicken DT40 cells), the C-SP will be cleaved with the help of the mannose; however, instead of getting attached to a GPI anchor, the unanchored protein will be secreted [9]. As a result, deficiencies in the GPI anchor synthesis pathway can manifest in either the degradation (PIG-A mutation in mammalian cells) or the secretion (PIG-O mutation in chicken cells) of the unanchored proteins.

Taking advantage of the fact that PIG-A functional mutations can be easily detected by the absence of GPIanchored proteins on the cell surface, in vivo and in vitro PIG $A$ mutational assays have been developed, whereby the loss of cell surface GPI-anchored proteins is detected via antibody staining [10-16]. The in vivo Pig-a mutation assay has been used in many laboratories for basic science as well as regulatory science [17-26]. Now, a new Organization for Economic Cooperation and Development (OECD) test guideline in vivo Pig-a mutation assay are under preparations [17]. However, as with any antibody-based assay, this method is contingent upon the availability of sensitive and specific antibodies. In the current study, we have bypassed the limitations of antibody-based assays by constructing a GPI-sensor in which the fluorescent proteins GFP and mCherry were fused to the signal peptides at the $\mathrm{N}$ - and C-termini. The presence of GFP and mCherry on the cell surface is dependent on a functional GPI anchor synthesis pathway, and loss-of-function mutations in PIG- $A$ lead to the absence of fluorescent signal. This modified, GPI-sensor PIG-A mutation assay should be widely relevant for many applications, in both mammalian cells (for PIG-A mutations) and in the widely-used chicken DT40 cell lines (for PIG-O mutations).

\section{Materials and methods Chemicals and reagents}

The following chemicals and reagents were used in the study: Methyl methanesulfonate [66-27-3] (MMS) (Sigma), polybrene [28728-55-4], puromycin [53-79-2] (Sigma), TRIzol ${ }^{\circ}$ RNA Isolation Reagent (Invitrogen), RPMI 1640 culture medium (Invitrogen), penicillin/ streptomycin (Invitrogen), fetal bovine serum (Atlanta Biologicals), iScript ${ }^{\text {tx }}$ cDNA Synthesis Kit (Bio-Rad Laboratories, Inc.), Taq DNA Polymerase (New England Biolabs.), TransIT ${ }^{\oplus}-293$ transfection reagent (Mirus Bio LLC) and Fixable Viability Stain 450(FVS450) (BD Biosciences).

\section{Lentiviral-mediated CDNA expression}

The GFP and $m$ Cherry genes were inserted into the pUltra vector, along with the translocation signal sequence of acrosin on the $5^{\prime}$ end and the CD90 GPI anchor signal sequence in the $3^{\prime}$ end, all under the control of the hUBC promoter [27]. Lentiviral particles were prepared and target cells were infected according to a standard protocol, as previously used in our lab [28]. Briefly, $2 \mu \mathrm{g}$ of pUltra GPI-GFP or GPI-mCherry, $2 \mu \mathrm{g}$ of pREV, $2 \mu \mathrm{g}$ of pGag/Pol and $1 \mu \mathrm{g}$ of pVSVG were transfected into HEK293T cells in a $6 \mathrm{~cm}$ dish using the TransIT ${ }^{\circ}-293$ transfection reagent. Cell culture medium was changed $24 \mathrm{~h}$ later, and the supernatant containing lentiviral particles was collected 2 days after transfection. TK6 cells were infected with lentivirus in the presence of $5 \mu \mathrm{g} / \mathrm{ml}$ polybrene, and after $24 \mathrm{~h}$, fresh medium was replaced. Two to three days post-transduction, the expression of the fluorescent proteins was detected via fluoresce microscope and flow cytometry.

\section{Cell culture}

Human B-lymphoblastoid TK6 cells were maintained in our lab and were cultured in RPMI-1640 medium supplemented with $10 \%$ fetal bovine serum and $1 \%$ penicillin/streptomycin. HEK293T cells were purchased from the Lineberger Comprehensive Cancer Center at the University of North Carolina at Chapel Hill and were cultured in DMEM medium supplemented with Lglutamine, $10 \%$ fetal bovine serum and $1 \%$ penicillin/ streptomycin. All cells were maintained at $37^{\circ} \mathrm{C}$ with $5 \% \mathrm{CO} 2$.

\section{Flow cytometry for cell sorting and detection}

GFP and mCherry double positive TK6 cells were sorted using a FACSAria II (BSL2) (UNC Flow Cytometry Core Facility) using a standard protocol. Briefly, cells were 
harvested and washed with PBS and then placed in sorting buffer (HEPES-buffered HBSS with $1 \%$ BSA) at $10^{7}$ cells $/ \mathrm{ml}$. Target cells were sorted into collection buffer (HEPES-buffered RPMI 1640 medium with 10\% fetal bovine serum).

Flow cytometry analyses were performed with an LSRFortessa (BSL2) (UNC Flow Cytometry Core Facility). Cells were collected and washed with PBS. After staining with the BD Horizon ${ }^{\mathrm{Tx}}$ Fixable Viability Stain 450 (FVS450) for $15 \mathrm{~min}$ at room temperature using a standard protocol, the cells were washed with PBS and filtered before flow cytometry analyses. Excitation and emission detection wavelengths of the fluorescence were the following: FITC (488-530/30 nm), mCherry (561$610 / 20 \mathrm{~nm})$, FVS450 (405-450/50 nm). Samples were analyzed at $\sim 8000$ events/second. More than $10^{6}$ cells were collected in order to detect GPI(-) frequency.

\section{Spiking experiment}

Spiked cell samples were prepared by mixing $0,10,20$, 40, $80 \mathrm{GPI}(-)$ cells into every $10^{6}$ freshly re-populated GPI $(+)$ cells.

\section{Determination of the phenotypic expression time for TK6 cells}

To determine the phenotypic expression time for TK6 cells, GPI $(+)$ cells were treated with 12 and $24 \mu \mathrm{M}$ MMS for $24 \mathrm{~h}$, and GPI(-) cells were detected via flow cytometry every 2 days from day 8 to day 14 .

\section{MMS treatment}

$3 \times 10^{6}$ cells were incubated in $10 \mathrm{ml}$ medium with MMS. After $24 \mathrm{~h}$ of exposure, cells were washed with PBS and $2 \times 10^{6}$ cells were subcultured every other day into $25 \mathrm{ml}$ of medium.

\section{A}

\section{Fluorescent} Protein

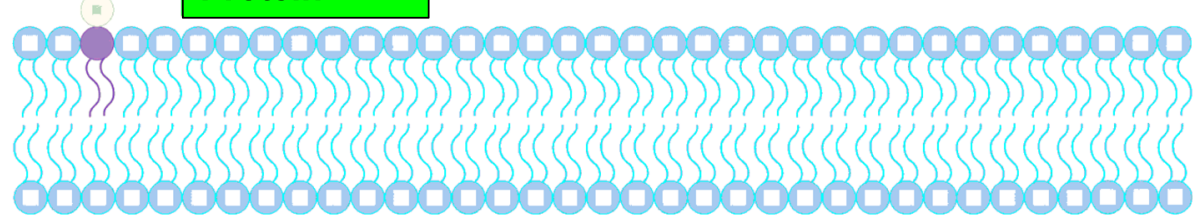
$\uparrow$ With the help of PIGs GFP is In the absence of GPI anchor GFP anchored to GPI anchor on cell membrane will be eliminated. No GFP will be targeted on cell membrane

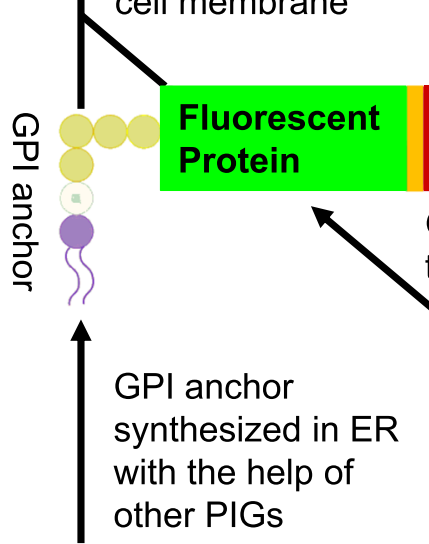

PIG-A

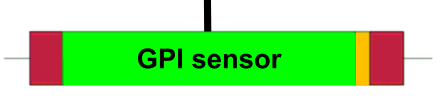

No GPI anchor synthesized in ER in the absence of functional PIG-A

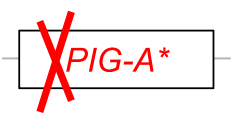

B

Fig. 1 Fluorescent GPI-sensor PIG-A mutation assay schematic and GPI-anchored protein precursor structure. a GPI-sensor PIG-A mutation assay schematic. b GPl-anchored protein precursor structure 


\section{PIG-A sequencing}

cDNA was prepared as previously described [28]. Briefly, total RNA was isolated by using TRIzol ${ }^{\circ}$ RNA Isolation Reagent following the manufacture's protocol. The same amount of total RNA was used for reverse transcription using the iScript $^{\text {tix }}$ cDNA Synthesis Kit following the manufacture's protocol. Taq DNA polymerase and the following primers were used to amplify PIG-A: F1: 5' > ggttgctctaagaactgatgtc $\quad<3^{\prime} ; \quad \mathrm{R} 1: \quad 5^{\prime}>$ atcatgccttctaaatgggtc $<3^{\prime} ; \mathrm{F} 2: 5^{\prime}>{\text { agcttctaaccgtgtctctt }<3^{\prime} ; \mathrm{R} 2: 5^{\prime}>}^{\prime}>$ cccccaaaagcaaggttatt $<3^{\prime}$. For F1\&R1, $64^{\circ} \mathrm{C}$ was used for the annealing temperature, and for $\mathrm{F} 2 \& \mathrm{R} 2,56^{\circ} \mathrm{C}$ was used. The primer set F1 and R3: $5^{\prime}>$ tcttacaatctaggcttccttc $<3^{\prime}$ was used for sequencing. The PIG-A gene sequence from freshly re-populated $\mathrm{GPI}(+)$ cells was used as a wild-type reference.

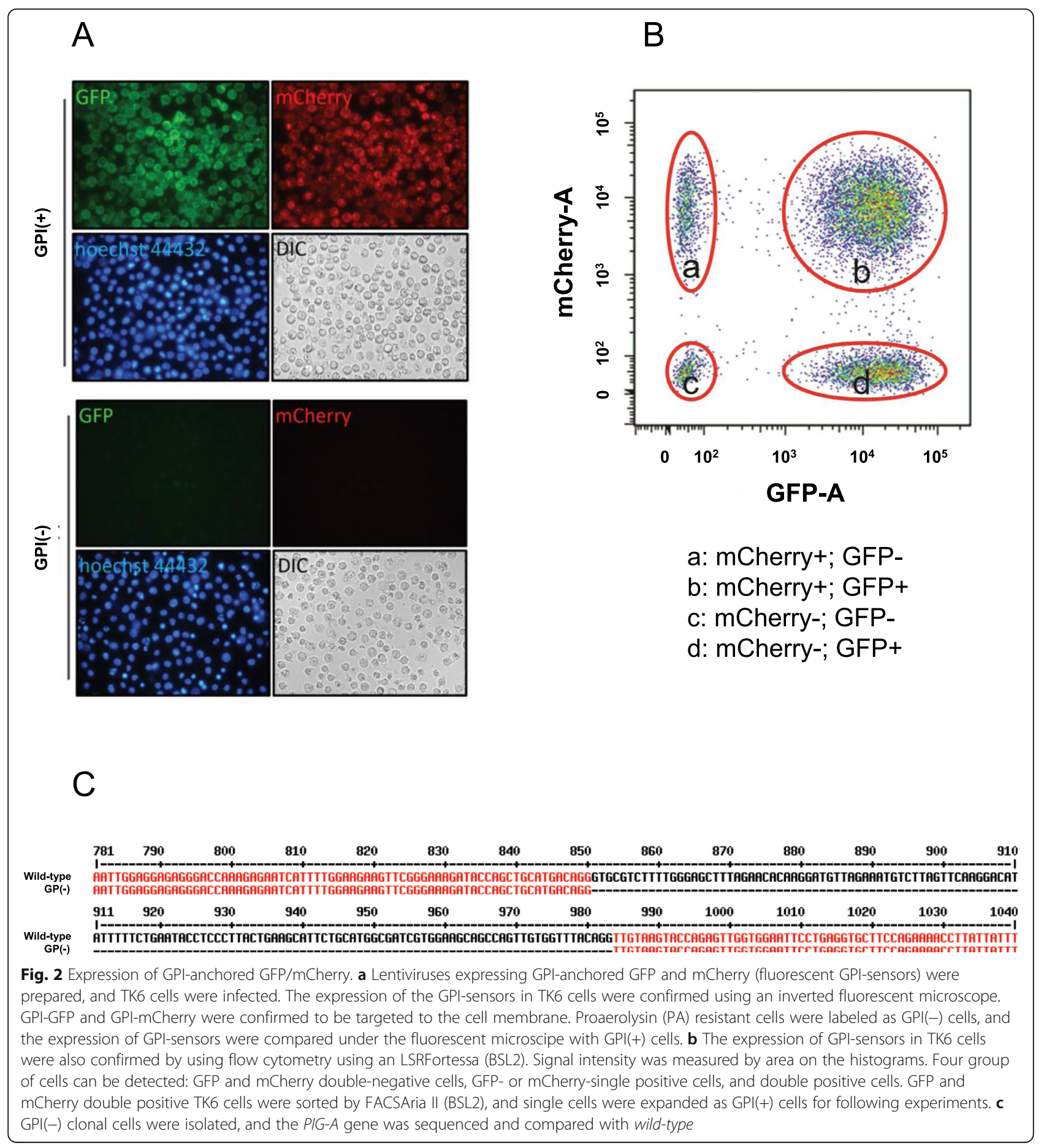




\section{Results}

\section{Design and expression of fluorescent sensors on the cell} surface

The conventional PIG- $A$ mutation assay involves measuring levels of endogenous GPI-anchored proteins on the cell surface using antibodies specific for these proteins [12-16]. In this study, we wanted to determine whether we could artificially express and tether proteins on the cell surface via a GPI anchor. To address this question, we designed constructs for GPI-anchored fluorescent proteins (Fig. 1). The N-terminus of the fluorescent protein was tagged with a hydrophobic signal peptide (N-SP) that targeted the fluorescent protein to the endoplasmic reticulum lumen. The C-terminal signal peptide (C-SP) was also added for the addition of the GPI-anchor via post translational modification [8]. Successful detection of the resulting mature fluorescent protein at the cell surface indicates a functional, intact GPI-anchor synthesis pathway. On the other hand, if there is any malfunction in the GPIanchor synthesis pathway, fluorescent proteins would either be degraded or secreted outside of cells such that no fluorescence at the cell surface would be observed.

Theoretically, when using a GPI-sensor to detect the presence of functional mutations in the PIG- $A$ gene, a mutation in the sensor itself could interfere with the assay. To address this potential issue, we expressed two fluorescent sensors in each cell. If the fluorescent sensor mutation rate is similar to the PIG- $A$ spontaneous mutation rate, which is around $10^{-6}$, the ratio of cells that develop mutations in both fluorescent sensors will be around $10^{-12}$, which is a rate that can deemed as negligible in the PIG- $A$ mutation assay. In our assay, we co-expressed GPI-GFP and GPImCherry in our cells, and only double negative cells were deemed to have a PIG- $A$ mutation.

We constructed GPI-anchored GFP and GPI-anchored mCherry lentiviral expression vectors and infected TK6 cells with the GPI-anchored GFP and GPI-anchored mCherry lentiviruses using a standard protocol [28]. To confirm the expression of GFP and mCherry, we examined the cells infected with the lentiviruses under a fluorescent microscope and found that both GFP and mCherry were present on the cell surface (Fig. 2a). Next, we determined if we could separate GFP and mCherry double-positive cells from single-positive and double-negative cells by flow cytometry using an LSRFortessa (BSL2). The four cell populations were clearly separated (Fig. 2b, Groups (a)-(d)). We further sorted GFP and mCherry double-positive cells (Fig. 2b, Group (b)) by FACSAria II (BSL2) and cultured them for further experiments as GPI(+) cells.

\section{Fluorescent signals are absent in cells carrying PIG-A mutation}

To determine if the expression of GPI-GFP or mCherry on the cell surface is indeed dependent on intact PIG-A

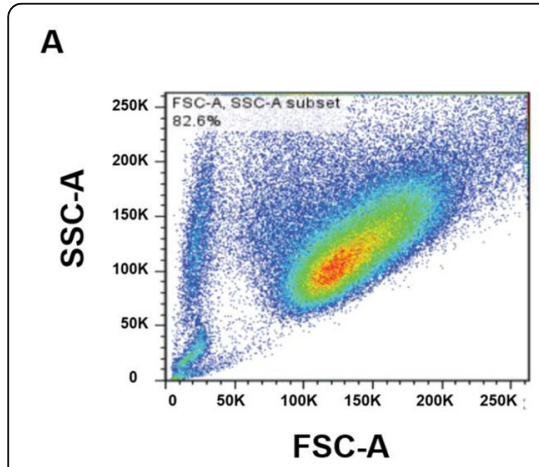

D

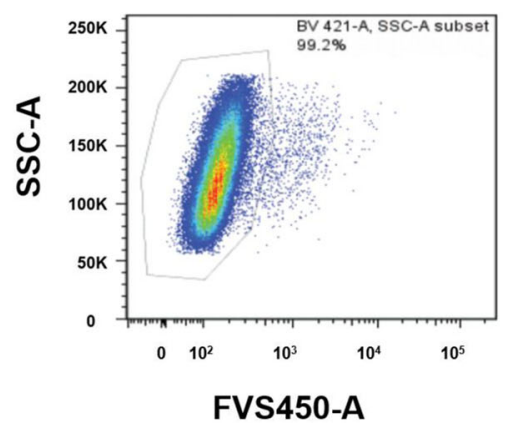

B

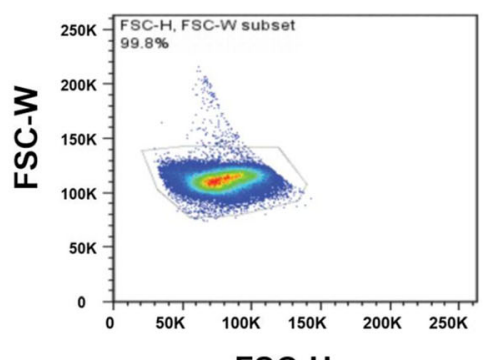

FSC-H

E

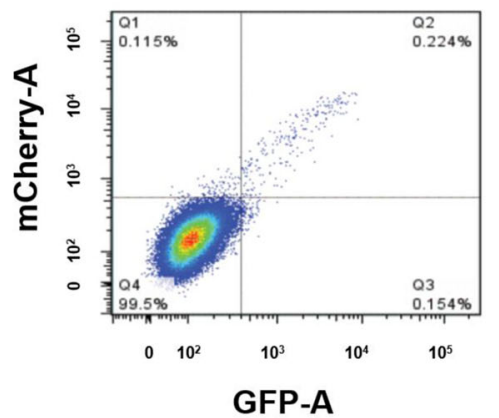

C

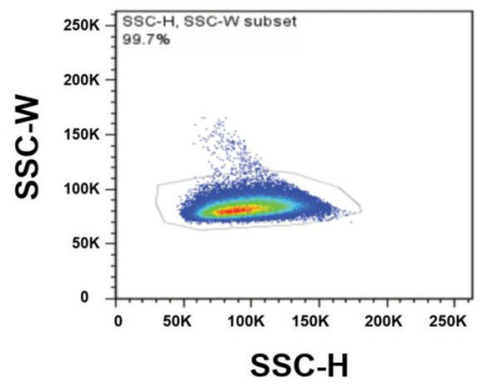

$\mathbf{F}$

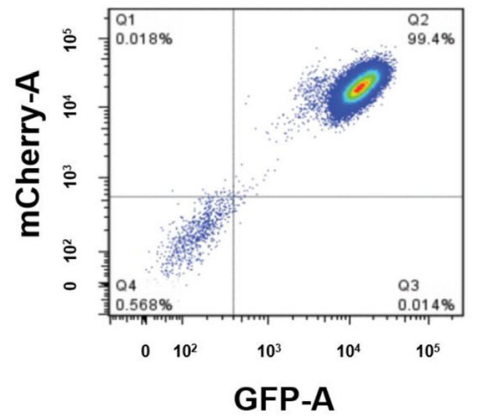

Fig. 3 Flow cytometric analysis of GPI(-) cell frequency. Flow cytometry was performed using an LSRFortessa (BSL2). a Cells were collected by FSC/SSC, b, c Single cells were collected via FSC-W/FSC-H and SSC-W/SSC-H. d Dead cells were eliminated by FVS450 staining. GPI(-) (e) and $\mathrm{GPI}(+)(\mathbf{f})$ cell numbers were measured, and spontaneously mutated cells were isolated in (f) Q4 
function, we first generated cells carrying PIG- $A$ mutations. We treated TK6 cells with proaerolysin (PA), which kills off cells with GPI-anchored proteins on the cell surface (i.e. cells without PIG- $A$ mutations). The remaining PA-resistant cells are those that have spontaneous PIG-A mutations [3]. We infected wild-type and PIG-A mutant cells with the GPI-GFP and mCherry lentiviruses and examined the fluorescent signal. We found that, unlike wild-type cells, no fluorescent signal could be detected in PIG-A mutant cells (Data not shown). To eliminate the possibility that a PIG-A deficiency could suppress viral infection, we treated $\mathrm{GPI}(+)$ cells that initially expressed both GFP and mCherry on the cell surface with PA and selected the resistant cells that developed spontaneous PIG-A mutations. We found that GFP and mCherry were no longer expressed on the surface of these cells (Fig. 2a). These results suggest that in the absence of GPI anchor synthesis (i.e. in cells with a PIG- $A$ mutation), GPI-GFP/mCherry is degraded or secreted, and there is no fluorescent signal maintained in the cells. We sorted these cells and maintained them as GPI(-) cells for further experiments.

To confirm the presence of a mutation in the PIG-A gene in GFP and mCherry double negative cells, we sorted the cells by FACSAria II and cultured single clone cells. Ten clonal populations of cells were exposed to PA and were found to be resistant, indicating the presence of mutation in PIG-A. Additionally, sequencing the PIG$A$ gene of those clonal cells identified the same deletion mutation in all ten clones (Fig. 2c).

\section{Reduction of a spontaneous PIG-A mutation by repopulation}

The gating procedure for flow cytometry analysis using the LSRFortessa is shown in Fig. 3. Cells were collected by FSC/SSC gate scatter (Fig. 3a), and single cells were collected by FSC-W/FSC-H and SSC-W/ SSC-H (Fig. 3b and c). Dead cells were excluded via FVS450 staining (Fig. 3d). GPI(-) cells were used as a negative control (Fig. 3e). GPI (+) cells were measured,

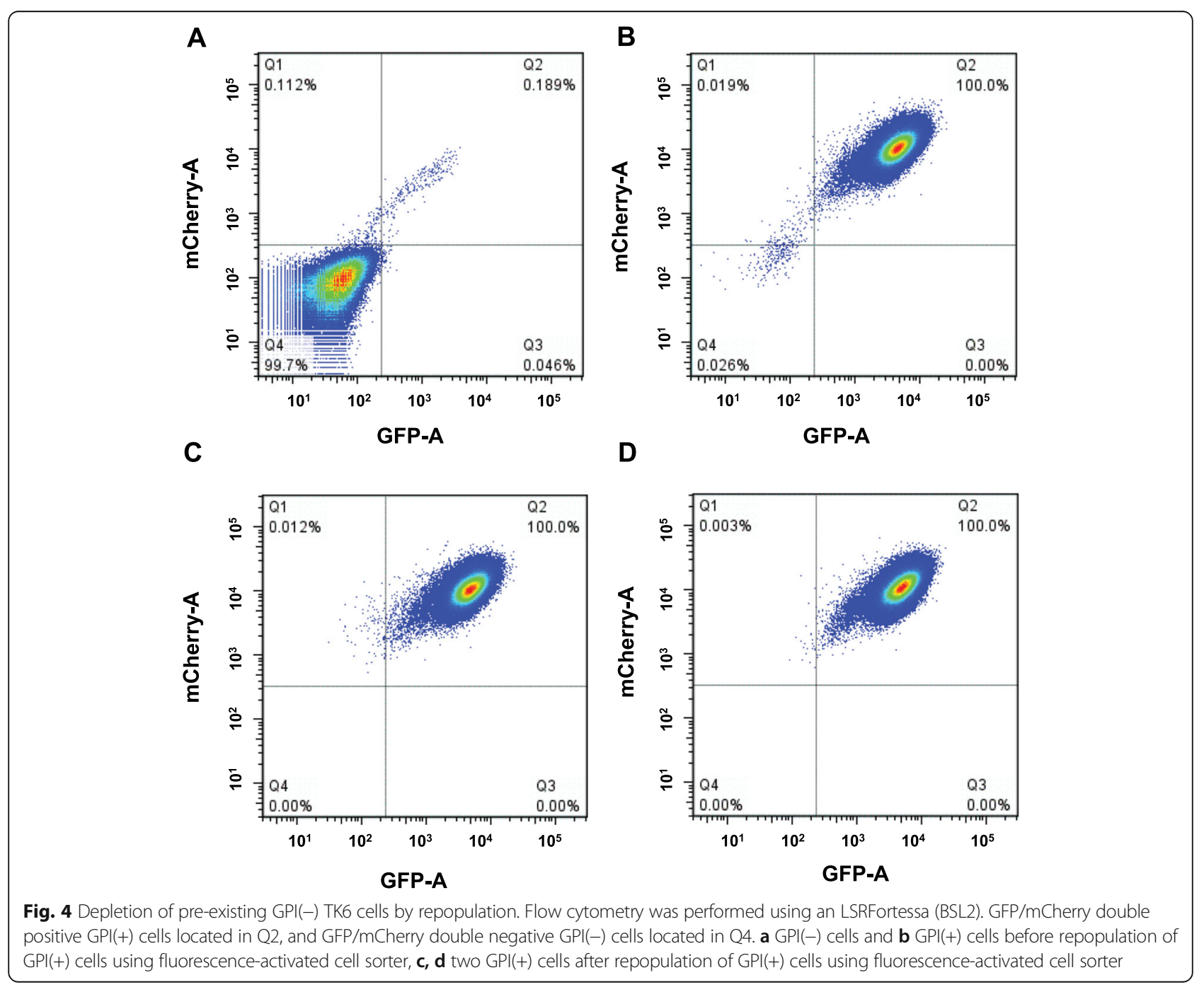


and wild-type cells (Fig. 3f, Q2) and spontaneous mutation cells (Fig. 3f Q4) were separated.

There was a small amount of cells carrying spontaneous PIG-A mutations which can accumulate over time or in the process of cell expansion (Fig. 4b). The presence of these GPI(-) cells can reduce the consistency and sensitivity of this mutation assay, so it is necessary to exclude these cells. To accomplish this, we repopulated 4000-5000 cells (based on an actual negative cell rate) in multiple dishes. Afterward, we obtained some dishes with low preexisting GPI(-) cells. Two examples of repopulated cells were shown Fig. $4 \mathrm{c}$ and d.

\section{Detection of rare GPI(-) cells}

It is necessary that we are able to detect rare mutations from a large pool of wild type cells. To determine the sensitivity of this assay, we performed spiking experiment, in which we artificially mixed a few GPI(-) cells with a large amount of $\mathrm{GPI}(+)$ cells and examined if similar amount of GPI(-) cells could be detected using flow cytometry. We found that the ratio of spiked GPI(-) cells correlated linearly with the mutation rate (Fig. 5), suggesting that this GPI-sensor PIG- $A$ mutation assay is sufficient to recover and quantify vary rare GPI(-) cells.

\section{Optimization of GPI(-) phenotypic expression period}

Next, we determined the optimal GPI(-) phenotypic expression period for TK6 cells for the GPI-sensor PIG-A mutation assay. The phenotypic expression period is the time required for mutated cells to have the wild-type PIG$A$ mRNA, protein and existing GPI-anchored proteins to be either degraded or diluted to allow for the manifestation of the mutation phenotype. To determine the optimal GPI(-) phenotypic expression period, we exposed TK6 cells with water, $12 \mu \mathrm{M}$ or $24 \mu \mathrm{M}$ of MMS for $24 \mathrm{~h}$ before collecting samples. Frequency of GPI(-) cells was measured every two days by flow cytometry from day 8 to day 14 (Fig. 6). As expected, cells treated with water did not show any significant change in the frequency of GPI(-) cells (Fig. 6a, blue line). Exposure of cells to either $12 \mu \mathrm{M}$ or $24 \mu \mathrm{M}$ of MMS showed an initial increase in the ratio of GPI(-) cells, which peaked at around day 12 , after which the mutational frequency plateaued. Thus, the phenotypic expression period for TK6 cells for GPIsensor PIG-A mutation assay was set at 12 days.

\section{Fluorescent sensor assay effectively detects mutation in cells post-MMS exposure}

Finally, we measured the ratio of cells harboring PIG-A mutations and cell proliferation as a result of MMS exposure. To do this, GPI(+) TK6 cells were treated with water, $12 \mu \mathrm{M}$ or $24 \mu \mathrm{M}$ MMS. Cell numbers were counted every two days, and relative increases in cell counts were calculated accordingly. The PIG-A mutation rate was measured on day 12 after MMS exposure. We found that cells treated with $24 \mu \mathrm{M}$ MMS contained the highest ratio of $\operatorname{GFP}(-)$ cells compared to control or $12 \mu \mathrm{M}$ MMS treatment (Fig. 6b). At the same time, we found that MMS treatment suppressed cell proliferation. On day 12, the total number of cells after $24 \mathrm{~h}$ of MMS treatment was only $25 \%$ that of control treatment.

\section{Discussion}

In the current study, we have successfully established a novel PIG-A mutation assay that takes advantage of the

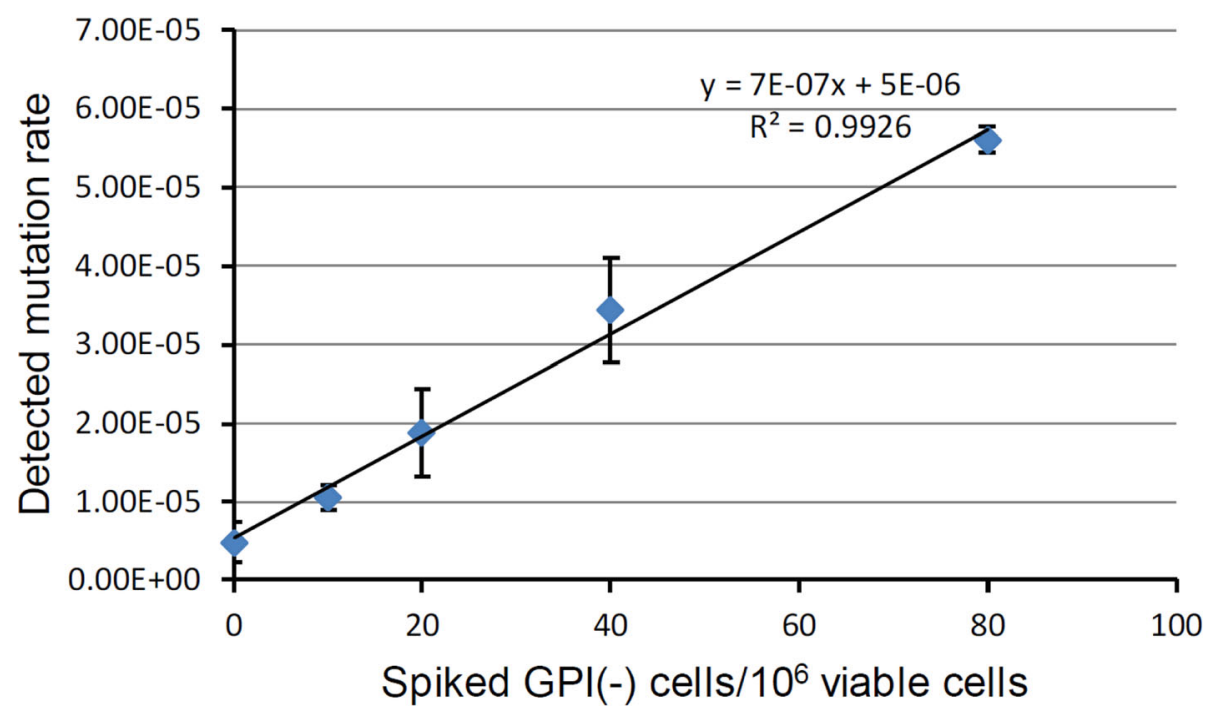

Fig. 5 Recovery of spiked GPI(-) TK6 cells via flow cytometry. 0, 10, 20, 40 and 80 GPI(-) cells were spiked into $1 \times 10^{6}$ repopulated GPI(+) cells, and the mutation rate $(\mathrm{GPI}(-)$ cells) were detected via flow cytometry. Shown are mean value \pm SD for three independent samples for each spiking level 


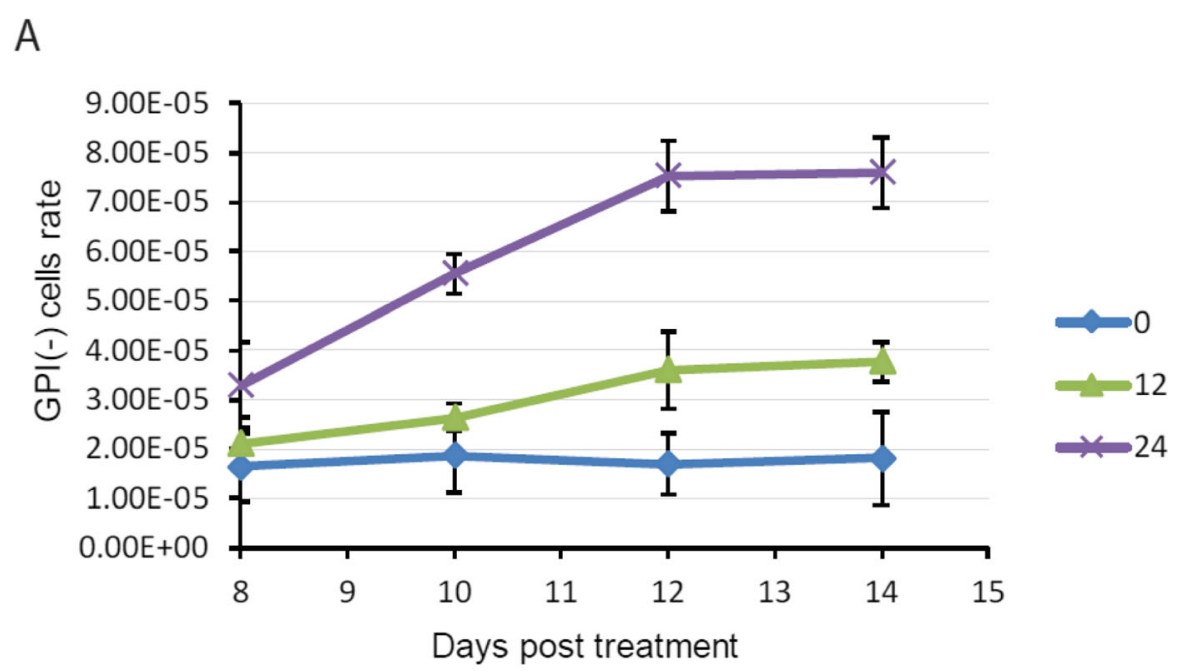

B

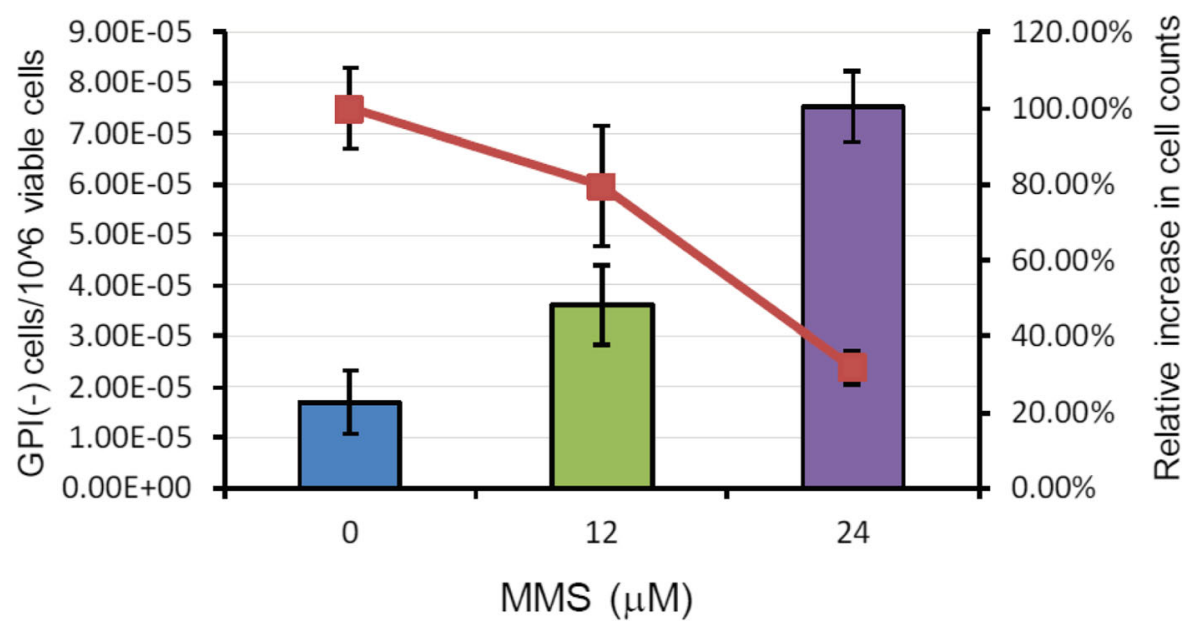

Fig. 6 Detection of mutations after MMS treatment. a GPI(-) phenotypic expression time for TK6 cells. Cells were treated with 0,12 or $24 \mu \mathrm{M}$ MMS for $24 \mathrm{~h}$, washed and cultured in fresh medium. GPI(-) frequency was detected by flow cytometry on days $8,10,12$ and 14 after exposure. Shown are mean value \pm SD for three independent samples. GPI(-) cells frequency after $24 \mu \mathrm{M}$ MMS exposure (b). Bars represent the GPI(-) frequency and lines represent relative increases in cells counts

GPI anchor synthesis pathway. We generated GPI-GFP and GPI-mCherry double expressing TK6 wild-type cells and confirmed their membrane localization via fluorescent microscopy. The absence of GFP and mCherry fluorescence in PIG-A mutation cells indicated the lack of cell surface GPI-anchored protein expression, which was confirmed by PA resistance and PIG-A sequencing. A spiking experiment shows that our fluorescent GPIsensor is sufficient to detect very low frequency of GPI(-) negative cells. Finally, we exposed TK6 cells to MMS, determined the phenotypic expression time, and examined genotoxicity induced by MMS using our newly established fluorescent GPI-sensor PIG- $A$ mutation assay.
Conventional PIG-A mutation assays are contingent upon antibodies that are sufficiently sensitive and specific for the detection of endogenous GPI-anchored proteins. This antibody-based assay can be expensive and time-consuming. In some conditions, suitable antibodies may not be available for certain cell lines. To avoid the caveats of antibody staining which may result nonspecific labelling or inconsistent measurements, we engineered artificial GPI-anchored fluorescent proteins, GPI-GFP and GPI-mCherry, that are tethered to the membrane. Mutations in PIG-A lead to a deficiency in GPI anchor synthesis and, consequently, an absence of fluorescent signal. Our fluorescent GPI-sensor PIG-A mutation assay completely eradicates the need for 
antibody staining, which not only saves time and resources but also extends the use of the PIG- $A$ mutation assay to a broader field of cells types and species. In theory, any eukaryotic cell which has one copy of any critical GASP (where one functional mutation in that GASP will cause phenotype changes) will be suitable for our fluorescent GPI-sensor mutation assay.

For example, we previously attempted to establish a flow cytometry-based PIG-O mutation in DT40 cells [3]; however, we were unable to find suitable antibodies or a specific dye to distinguish GPI(-) cells from GPI(+) cells. Recently, we applied our fluorescent GPI-sensor assay to the DT40 PIG-O mutation assay. We found that our GPI-sensor is compatible with DT40 cells, and our preliminary data show successful expression of the GPIsensor in DT40 cells (data not shown). With minor optimizations, our fluorescent GPI-sensor should also work with the DT40 PIG-O mutation assay.

To confirm the presence of mutations in the PIG-A gene, we sequenced ten clonal spontaneously mutated PIG(-) cells. To our surprise, all of the clones showed the same deletion mutation as PIG(+) cells. This may be due to the possibility that the mutation occurred at a relatively early stage when we repopulated the cells.

Of note, it has previously been reported that in TK6 cells, there is a hemizygous deletion at the 17p12-p22 (275,712 bp) locus, which covers the $5^{\prime}$ region of the PIG$L$ gene [29]. This means that in TK6 cells, a single functional mutation in PIG-A or PIG- $L$ will cause a loss of GPI anchor synthesis function. In the case that a mutation in the PIG- $A$ gene is not detected, we will have to sequence $P I G-L$ in order to study the mutation spectrum.

\section{Conclusions}

In this study, we developed a modified PIG-A mutation assay utilizing GPI-anchored fluorescent protein expression that eliminates need for antibody-based staining. This GPI-sensor PIG-A mutation assay is compatible with many mammalian and vertebrate cells, such as DT40 cells, and should be widely applicable for accurate and efficient testing of genotoxicity.

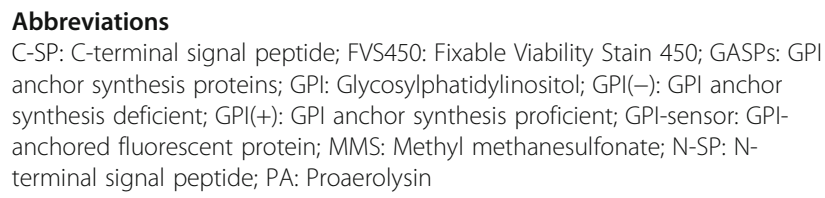

\section{Acknowledgments}

We would like to thank James A. Swenberg for supporting this study. We are grateful to Peter Howard, University of Saskatchewan, Saskatchewan, Canada, for providing proaerolysin.

\section{Authors' contributions}

Conceived and designed the experiments: JN. Performed the experiments: XT. Analyzed the data: XT, YC. Wrote the paper: XT, YC, JN. All authors read and approved the final manuscript

\section{Funding}

This work was supported by JSPS KAKENHI Grant Numbers $17 \mathrm{H} 07027$ and the Health and Labour Science Research Grants from Ministry of Health, Labour and Welfare of the Japanese Government.

\section{Availability of data and materials \\ Not applicable.}

Ethics approval and consent to participate

Not applicable.

\section{Consent for publication}

Not applicable.

\section{Competing interests}

The authors declare that they have no competing interests.

\section{Author details}

'Department of Environmental Sciences and Engineering, University of North Carolina at Chapel Hill, Chapel Hill, NC, USA. ${ }^{2}$ Department of Neurology, UNC Neuroscience center, School of Medicine, University of North Carolina at Chapel Hill, Chapel Hill, North Carolina, USA. ${ }^{3}$ Laboratory of Laboratory Animal Science, Graduate School of Life and Environmental Biosciences, Osaka Prefecture University, Izumisano, Osaka, Japan.

Received: 9 October 2019 Accepted: 14 November 2019

Published online: 10 December 2019

\section{References}

1. Ames BN. Identifying environmental chemicals causing mutations and cancer. Science. 1979;204(4393):587-93.

2. Miyata T, Takeda J, lida Y, Yamada N, Inoue N, Takahashi M, et al. The cloning of PIG-A, a component in the early step of GPI-anchor biosynthesis. Science. 1993;259(5099):1318-20.

3. Nakamura J, Gul H, Tian X, Bultman SJ, Swenberg JA. Detection of PIGOdeficient cells using proaerolysin: a valuable tool to investigate mechanisms of mutagenesis in the DT40 cell system. PLoS One. 2012;7(3):e33563.

4. Bessler M, Hillmen P, Longo L, Luzzatto L, Mason PJ. Genomic organization of the X-linked gene (PIG-A) that is mutated in paroxysmal nocturnal haemoglobinuria and of a related autosomal pseudogene mapped to 12q21. Hum Mol Genet. 1994;3(5):751-7.

5. Takeda J, Miyata T, Kawagoe K, lida Y, Endo Y, Fujita T, et al. Deficiency of the GPI anchor caused by a somatic mutation of the PIG-A gene in paroxysmal nocturnal hemoglobinuria. Cell. 1993;73(4):703-11.

6. Brodsky RA, Hu R. PIG-A mutations in paroxysmal nocturnal hemoglobinuria and in normal hematopoiesis. Leuk Lymphoma. 2006;47(7):1215-21.

7. Orlean P, Menon AK. Thematic review series: lipid posttranslational modifications. GPI anchoring of protein in yeast and mammalian cells, or: how we learned to stop worrying and love glycophospholipids. J Lipid Res. 2007:48(5):993-1011.

8. Mayor S, Riezman H. Sorting GPI-anchored proteins. Nat Rev Mol Cell Biol. 2004;5(2):110-20.

9. Murakami Y, Kanzawa N, Saito K, Krawitz PM, Mundlos S, Robinson PN, et al. Mechanism for release of alkaline phosphatase caused by glycosylphosphatidylinositol deficiency in patients with hyperphosphatasia mental retardation syndrome. J Biol Chem. 2012;287(9):6318-25.

10. Peruzzi B, Araten DJ, Notaro R, Luzzatto L. The use of PIG-A as a sentinel gene for the study of the somatic mutation rate and of mutagenic agents in vivo. Mutat Res. 2010:705(1):3-10.

11. Araten DJ, Nafa K, Pakdeesuwan K, Luzzatto L. Clonal populations of hematopoietic cells with paroxysmal nocturnal hemoglobinuria genotype and phenotype are present in normal individuals. Proc Natl Acad Sci U S A. 1999:96(9):5209-14.

12. Gollapudi BB, Lynch AM, Heflich RH, Dertinger SD, Dobrovolsky VN, Froetschl $R$, et al. The in vivo pig-a assay: A report of the international workshop on Genotoxicity testing (IWGT) workgroup. Mutat Res Genet Toxicol Environ Mutagen. 2015;783:23-35.

13. David R, Talbot E, Allen B, Wilson A, Arshad U, Doherty A. The development of an in vitro pig-a assay in L5178Y cells. Arch Toxicol. 2018;92(4):1609-23.

14. Bemis JC, Avlasevich SL, Labash C, McKinzie P, Revollo J, Dobrovolsky VN, et al. Glycosylphosphatidylinositol (GPI) anchored protein deficiency serves 
as a reliable reporter of pig-a gene mutation: support from an in vitro assay based on L5178Y/Tk(+/-) cells and the CD90.2 antigen. Environ Mol Mutagen. 2018:59(1):18-29.

15. Kruger $\mathrm{CT}$, Hofmann M, Hartwig A. The in vitro PIG-A gene mutation assay: mutagenicity testing via flow cytometry based on the glycosylphosphatidylinositol (GPI) status of TK6 cells. Arch Toxicol. 2015; 89(12):2429-43.

16. Araten DJ, Golde DW, Zhang RH, Thaler HT, Gargiulo L, Notaro R, et al. A quantitative measurement of the human somatic mutation rate. Cancer Res. 2005;65(18):8111-7.

17. Chikura S, Kimoto T, Itoh S, Sanada H, Muto S, Horibata K. Standard protocol for the total red blood cell pig-a assay used in the interlaboratory trial organized by the mammalian mutagenicity study Group of the Japanese Environmental Mutagen Society. Genes Environ. 2019;41:5.

18. Horibata K, Ukai A, Ogata A, Nakae D, Ando H, Kubo Y, et al. Absence of in vivo mutagenicity of multi-walled carbon nanotubes in single intratracheal instillation study using F344 gpt delta rats. Genes Environ. 2017;39:4.

19. Kimoto T, Horibata K, Chikura S, Hashimoto K, Itoh S, Sanada H, et al. Interlaboratory trial of the rat pig-a mutation assay using an erythroid marker HIS49 antibody. Mutat Res Genet Toxicol Environ Mutagen. 2013; 755:126-34.

20. Itoh S, Nagata M, Hattori C, Takasaki W. In vivo mutagenicity of ethyl Methanesulfonate detected by pig-a and PIGRET assays. Genes Environ. 2014;36:174-8

21. Sanada H, Okamoto M, Ohsumi T, Nakamura T. Evaluation for a mutagenicity of 4,4'-Methylenedianiline on hematopoietic cells by a pig-a gene mutation assay in rats. Genes Environ. 2014;36:179-85.

22. Muto S, Yamada K, Kato T, Iwase Y, Uno Y. Pig-a gene mutation and micronucleus induction in rat peripheral blood by methyl Methanesulfonate. Genes Environ. 2014;36:186-90.

23. Kimoto T, Chikura S, Suzuki-Okada K, Kobayashi X, Itano Y, Miura D, et al. The rat pig-a mutation assay in single and 28 day-repeated dose study of cyclophosphamide: the PIGRET assay can detect the in vivo mutagenicity earlier than the RBC pig-a assay. Genes Environ. 2014:36:191-8.

24. Kimoto T, Horibata K, Miura D, Chikura S, Okada Y, Ukai A, et al. The PIGRET assay, a method for measuring pig-a gene mutation in reticulocytes, is reliable as a short-term in vivo genotoxicity test: summary of the MMS/ JEMS-collaborative study across 16 laboratories using 24 chemicals. Mutat Res Genet Toxicol Environ Mutagen. 2016;811:3-15.

25. Dobrovolsky VN, Boctor SY, Twaddle NC, Doerge DR, Bishop ME, Manjanatha MG, et al. Flow cytometric detection of pig-a mutant red blood cells using an erythroid-specific antibody: application of the method for evaluating the in vivo genotoxicity of methylphenidate in adolescent rats. Environ Mol Mutagen. 2010;51(2):138-45.

26. Dertinger SD, Phonethepswath S, Weller P, Nicolette J, Murray J, Sonders P, et al. International pig-a gene mutation assay trial: evaluation of transferability across 14 laboratories. Environ Mol Mutagen. 2011:52(9):690-8.

27. Rhee JM, Pirity MK, Lackan CS, Long JZ, Kondoh G, Takeda J, et al. In vivo imaging and differential localization of lipid-modified GFP-variant fusions in embryonic stem cells and mice. Genesis. 2006;44(4):202-18.

28. Tian X, Patel K, Ridpath JR, Chen Y, Zhou YH, Neo D, et al. Homologous recombination and Translesion DNA synthesis play critical roles on tolerating DNA damage caused by trace levels of hexavalent chromium. PLoS One. 2016;11(12):e0167503.

29. Nicklas JA, Carter EW, Albertini RJ. Both PIGA and PIGL mutations cause GPIa deficient isolates in the Tk6 cell line. Environ Mol Mutagen. 2015;56(8): 663-73.

\section{Publisher's Note}

Springer Nature remains neutral with regard to jurisdictional claims in published maps and institutional affiliations.

Ready to submit your research? Choose BMC and benefit from:

- fast, convenient online submission

- thorough peer review by experienced researchers in your field

- rapid publication on acceptance

- support for research data, including large and complex data types

- gold Open Access which fosters wider collaboration and increased citations

- maximum visibility for your research: over $100 \mathrm{M}$ website views per year

At $\mathrm{BMC}$, research is always in progress.

Learn more biomedcentral.com/submissions 